IASSNS-HEP-97/2

UCLA $/ 97 / \mathrm{TEP} / 1$

Columbia/Math/97/1

\title{
STRONG COUPLING EXPANSIONS OF SU(N) SEIBERG-WITTEN THEORY*
}

\author{
Eric D'Hoker ${ }^{1}$ and D.H. Phong ${ }^{2}$ \\ ${ }^{1}$ Institute for Advanced Study \\ Princeton, NJ 08540, USA \\ and \\ Department of Physics \\ University of California, Los Angeles, CA 90024, USA \\ ${ }^{2}$ Department of Mathematics \\ Columbia University, New York, NY 10027, USA
}

\begin{abstract}
We set up a systematic expansion of the prepotential for $\mathcal{N}=2$ supersymmetric YangMills theories with $\mathrm{SU}(\mathrm{N})$ gauge group in the region of strong coupling where a maximal number of mutually local fields become massless. In particular, we derive the first nontrivial non-perturbative correction, which is the first term in the strong coupling expansion providing a coupling between the different $\mathrm{U}(1)$ factors, and which governs for example the strength of gaugino Yukawa couplings.
\end{abstract}

\footnotetext{
* Research supported in part by the National Science Foundation under grants PHY95-31023 and DMS-95-05399, and by the Harmon Duncombe Foundation.
} 


\section{INTRODUCTION}

The leading low energy behavior of $\mathcal{N}=2$ supersymmetric Yang-Mills theory is completely determined by the effective prepotential $\mathcal{F}$, as a function of the moduli of inequivalent vacuum states. The effective prepotential $\mathcal{F}$ is obtained in terms of the periods of a meromorphic Abelian differential $\mathrm{d} \lambda$, on a family of auxiliary Riemann surfaces parametrized by the moduli of vacua. This construction was pioneered in [1] for gauge group $\mathrm{SU}(2)$, extended in [2] to $\mathrm{SU}(\mathrm{N})$, to the inclusion of hypermultiplets in [3] and to other gauge groups in [4]. The power of this result resides in the fact that it is valid for all ranges of the coupling constant, including strong coupling. Of special interest is the dynamics of the theory close to the singularities in the moduli space of vacua; it is there that extra massless degrees of freedom appear.

At weak coupling, the Seiberg-Witten Ansatz reproduces the well-known perturbative expression, together with multi-instanton corrections. For gauge group $\mathrm{SU}(2)$, an expansion is readily derived from [1], and was found to agree with direct calculations in field theory in [5] to the orders available (up to field redefinitions). For gauge groups $\mathrm{SU}(\mathrm{N})$, $\mathrm{SO}(\mathrm{N})$ and $\mathrm{Sp}(\mathrm{N})$, (and any number of hypermultiplets in the fundamental repesentation of the gauge group), efficient methods for obtaining the instanton corrections to arbitrary order were developed and 1- and 2-instanton contributions evaluated explicitly in [6]. Geometrically, in the weak coupling region, the family of auxiliary Riemann surfaces degenerates to a product of two spheres joined by $\mathrm{N}$ thin tubes, thus producing an instanton expansion in terms of rational functions.

At strong coupling, singularities in the prepotential arise along subvarieties in the moduli space of vacua where certain magnetic magnetic monopoles or dyons become massless [1]. In view of the mass formula for BPS states, such massless states will appear whenever one (or more) of the periods of the differential $\mathrm{d} \lambda$ vanish. Geometrically, the strong coupling singularities will occur at the subvarieties in the moduli space of vacua where the auxiliary Riemann surfaces develop nodes.

The charge assignments of the magnetic monopoles or dyons that become massless at a given node, may be obtained directly from the Picard-Lefschetz theorem, and their associated monodromies were found to pass various physical consistency checks [1-4]. Their values may be used to obtain the logarithmically singular contributions to the prepotential, associated with the renormalization group behavior of the infrared free light dyons at those points. For gauge groups of rank at least 2, Argyres and Douglas [7] found that special subvarieties occur in the moduli space of vacua where mutually non-local dyons become massless simultaneously.

A more systematic analysis of the prepotential around strong coupling singularities has been difficult to obtain in general, however. One method makes use of the PicardFuchs equations for the periods of the meromorphic 1-form $\mathrm{d} \lambda$. It concisely reproduces 
the results for gauge group $\mathrm{SU}(2)$, and leads to explicit expressions for the quantum order parameters for $\mathrm{SU}(3)$ in terms of Appell functions, from which a strong coupling expansion may be obtained [8]. For gauge groups of rank 3, the associated Picard-Fuchs equations were derived only recently, and their complexity and sheer size suggest that it will be virtually impossible to generalize the results of [8] to gauge groups of higher rank.

In the present paper, we shall set up methods by which systematic strong coupling expansions of the prepotential at strong coupling singularities may be obtained directly from the geometry of the Riemann surface and the meromorphic 1-form. The key ingredients are as follows : (1) the prepotential is easily obtained from a renormalization group equation derived in [9-10], (2) the "beta-function", i.e. the right hand side of the renormalization group, is obtained by residue calculations on the small cycles of the Riemann surface.

We study theories with gauge group $\mathrm{SU}(\mathrm{N})$ and without hypermultiplets, but the generalization to other gauge groups and the inclusion of hypermultiplets is analogous. We shall concentrate on the region in the moduli space of vacua where the maximal number of mutually local dyons become massless (which is $\mathrm{N}-1$ for gauge group $\mathrm{SU}(\mathrm{N})$ ). These are precisly the strong coupling singularities for which the auxiliary Riemann surface degenerates to two spheres connected by $\mathrm{N}$ thin tubes, just as was the case for weak coupling, so that the prepotential may be expanded in a series of rational functions. These maximal strong coupling singularities are also the ones whose dynamics was studied in [11], in part in the limit of large numbers of colors. In particular, we derive the first non-trivial nonperturbative correction, which is the first term in the strong coupling expansion providing a coupling between the different $\mathrm{U}(1)$ factors, and which governs for example the strength of gaugino Yukawa couplings.

\section{EXPANSION AROUND CURVES OF MAXIMAL SINGULARITY}

The renormalized order parameters $a_{k}$, their duals $a_{D k}$ and the prepotential are defined by

$$
2 \pi i a_{k}=\oint_{A_{k}} \mathrm{~d} \lambda, \quad 2 \pi i a_{D k}=\oint_{B_{k}} \mathrm{~d} \lambda \quad \frac{\partial \mathcal{F}\left(a_{D}\right)}{\partial a_{D k}}=a_{k}
$$

in terms of the meromorphic Abelian differential $d \lambda$, given by

$$
d \lambda=\frac{x A^{\prime}(x) d x}{\sqrt{A(x)^{2}-4 \Lambda^{2 N}}}
$$

Here $A(x)$ is a polynomial of degree $\mathrm{N}$ in $x$, whose coefficients vary with the moduli of vacua, and $\Lambda$ is the renormalization scale. The associated Riemann surface (or curve) is of genus $N-1$ and is given by $y^{2}=A(x)^{2}-4 \Lambda^{2 N}$. Without loss of generality (but possible rescaling of $\Lambda$ ), the polynomial $A$ may be normalized so that $A(x)=x^{N}+u x^{N-2}+\mathcal{O}\left(x^{N-3}\right)$. 
We shall make use of the renormalization group equation for the prepotential found in $[8,9]$ and expressed here in terms of the dual order parameters :

$$
\sum_{k=1}^{N} a_{D k} \frac{\partial \mathcal{F}}{\partial a_{D k}}-2 \mathcal{F}=\frac{2 N}{2 \pi i} u\left(a_{D}\right)
$$

where $u$ is the expansion coefficient of $A(x)$ as defined above. This equation is extremely useful, because it now suffices to obtain the coefficient $u$ as a function of the periods $a_{D k}$. When the $B_{k}$ cycles degenerate, such a result is easily gotten from residue calculations, as was shown for the weak coupling regime in [10]. On the other hand, obtaining $\mathcal{F}$ directly from the the dual $A_{k}$ cycles, of (2.1), would involve complicated integrals over long cycles. Notice that in the above equation (2.3), the rôles of the renormalized order parameters $a_{k}$ and of their duals $a_{D k}$ may be interchanged.

\section{(a) The maximal singularity curves}

The strong coupling singularities at which a maximal number of mutually local magnetic monopoles or dyons become massless are characterized by curves $y^{2}=A_{0}(x)^{2}-4 \Lambda^{2 N}$ with a maximal number of distinct double zeros of $y^{2}$, and thus no zeros of order higher than 2. (Zeros of order 3 and above produce Argyres-Douglas singularities, and we shall not sudy those here.) As was shown in [11], these curves are given by the following (Chebychev) polynomials

$$
A_{0}(x)=2 \Lambda^{N} C_{0}\left(\frac{x}{2 \Lambda}\right) \quad C_{0}(z)=\cos (N \arccos (z))
$$

Since only $\Lambda^{2 N}$ entered the original problem, and $A_{0}(x)$ is a function involving only $\Lambda^{2}$, there are in general $N$ different solutions $A_{0}(x)$, associated with the $\mathrm{N}$ different roots of unity which can multiply $\Lambda$. All these solutions are physically equivalent and we shall restrict to the one for which $\Lambda$ is real.

The neighborhood of the maximal singularity curve may be parametrized by the $N-1$ parameter family of polynomials $P$ of degree $N-2$, whose coefficients are assumed to be small in units of $\Lambda$. Without loss of generality, we may render its coefficients dimensionless by scaling out suitable factors of $\Lambda$, similar to the form of (2.4). We then obtain the following parametrization of the neighborhood of the maximal singularity

$$
A(x)=A_{0}(x)+2 \Lambda^{N} P\left(\frac{x}{2 \Lambda}\right)
$$

Rescaling $x$ by a factor of $2 \Lambda$ so that it too becomes dimensionless, we have

$$
d \lambda=2 \Lambda \frac{x\left(C_{0}^{\prime}(x)+P^{\prime}(x)\right) d x}{\sqrt{\left(C_{0}(x)+P(x)\right)^{2}-1}}
$$


Henceforth, we shall measure the renormalized order parameters $a_{k}$ and their duals $a_{D, k}$ in units of $2 \Lambda$ and thus set $\Lambda=1 / 2$.

The double zeros of $C_{0}(x)$ are given by

$$
c_{k}=\cos \left(\frac{k \pi}{N}\right) \quad k=1, \cdots, N-1
$$

The coefficients of the polynomial $P(x)$ parametrize the way in which the $N-1$ double zeros are being approached. Generically, the effect of adding $P(x)$ in $(2.5)$ is to lift each double zero to a small branch cut in the double sheeted plane representation of the curve. We define the degenerating cycle $B_{k}, k=1, \cdots, N-1$, as the cycle that surrounds the branch cut created from the double zero $c_{k}$ once in the counterclockwise direction. The cycles $A_{k}$ can then be chosen to be any set of conjugate cyles, but we shall not need them here.

\section{(b) Expansion around degenerating cycles}

We assume that the coefficients of $P(x)$ are small compared to 1 , and that the curves $B_{k}$ are at a distance from $c_{k}$ of order a fraction of 1 , (and thus much larger than the coefficients of $P(x))$. Then, we may expand the period integrals for the $a_{D k}$ periods associated with the degenerating cycles $B_{k}$ in a convergent power series of $P(x)$. The radius of convergence for this expansion can be shown to be of order a fraction of 1 in these units.

To perform this expansion in an efficient way, it is most convenient to introduce an auxiliary parameter $\mu$ which interpolates between $C_{0}$ and $C_{0}+P$ by $C_{\mu}(x)=C_{0}(x)+\mu P(x)$. The period integrals now become

$$
a_{D k}=-\int_{0}^{1} d \mu \oint_{B_{k}} \frac{d x}{2 \pi i} \frac{P(x)}{\sqrt{C_{\mu}(x)^{2}-1}}=\sum_{m=0}^{\infty} a_{D k}^{(m)}
$$

with the expansion terms $a_{D k}^{(m)}$ given by

$$
a_{D k}^{(m)}=(-1)^{m+1} \frac{\Gamma\left(m+\frac{1}{2}\right)}{\Gamma\left(\frac{1}{2}\right) m !} \int_{0}^{1} d \mu \mu^{m} \oint_{B_{k}} \frac{d x}{2 \pi i} \frac{P(x)^{m+1}\left(\mu P(x)+2 C_{0}(x)\right)^{m}}{\left(C_{0}(x)^{2}-1\right)^{m+\frac{1}{2}}}
$$

We fix the square root sign by $\left(C_{0}(x)^{2}-1\right)^{\frac{1}{2}}=-i \sin (N \arccos x)$.

For low rank $N-1$ of the gauge group, the polynomial $P(x)$ will be of low degree, and one may use the coefficients of the polynomial as suitable classical parameters of moduli space of vacua. As soon as $N$ becomes larger, this parametrization is not convenient however. To identify a good parametrization, we analyze the first term $(m=0)$ in the above expansion. We introduce the very convenient notation

$$
P_{k}=P\left(c_{k}\right) ; \quad s_{k}=\sin \left(\frac{k \pi}{N}\right), \quad c_{k}=\cos \left(\frac{k \pi}{N}\right), \quad k=1, \cdots, N-1
$$


The first expansion term is given by

$$
a_{D k}^{(0)}=i(-)^{k} \frac{s_{k}}{N} P_{k}
$$

which indicates that convenient local coordinates of the moduli space of vacua around the maximal singularity are the values $P_{k}$ of $P(x)$ at the double points. We recover $P(x)$ with the help of the Lagrange interpolating formula giving the polynomial of degree $N-2$ in terms of its $N-1$ values $P_{k}$ at $N-1$ fixed points $c_{k}$

$$
P(x)=\sum_{k=1}^{N-1} \frac{C_{0}^{\prime}(x) P_{k}}{\left(x-c_{k}\right) C_{0}^{\prime \prime}\left(c_{k}\right)}
$$

To obtain useful formulas for the expansion coefficients, we take advantage of the fact that the factor of $C_{0}^{\prime}(x)$ almost eliminates the denominator in the integrand of (2.9). More precisely, we have

$$
\frac{C_{0}^{\prime}(x)}{\sqrt{C_{0}(x)^{2}-1}}=\frac{i N}{\sqrt{1-x^{2}}}
$$

We now introduce the functions $\Pi_{k}(x)$ and $G_{k}(x)$, which are analytic at $x=c_{k}$; we also define the functions $\Pi(x)$ and $G(x)$, which have simple poles at all $c_{k}$, and which we shall make use of later on.

$$
\begin{array}{ll}
\Pi_{k}(x)=\left(x-c_{k}\right) \Pi(x) & \Pi(x)=N^{2} \frac{P(x)}{C_{0}^{\prime}(x)}=-\sum_{l=1}^{N-1}(-)^{l} \frac{P_{l} s_{l}^{2}}{x-c_{l}} \\
G_{k}(x)=\left(x-c_{k}\right) G(x) & G(x)=N^{2} \frac{C_{0}(x)}{C_{0}^{\prime}(x)}=x N-\sum_{l=1}^{N-1} \frac{s_{l}^{2}}{x-c_{l}}
\end{array}
$$

In terms of these functions, the expansion coefficients $a_{D k}^{(m)}$ of $(2.9)$ are easily evaluated, and we obtain

$$
\begin{aligned}
& a_{D k}^{(m)}=-i \frac{\Gamma\left(m+\frac{1}{2}\right) / \Gamma\left(\frac{1}{2}\right)}{m ! N^{2 m+1}} \int_{0}^{1} d \mu \mu^{m} \oint_{B_{k}} \frac{d x}{2 \pi i} \frac{\left(1-x^{2}\right)^{-m-\frac{1}{2}}}{\left(x-c_{k}\right)^{2 m+1}} \Pi_{k}(x)^{m+1}\left(\mu \Pi_{k}(x)+2 G_{k}(x)\right)^{m} \\
& =-\left.i \frac{\Gamma\left(m+\frac{1}{2}\right) / \Gamma\left(\frac{1}{2}\right)}{m !(2 m) ! N^{2 m+1}} \int_{0}^{1} d \mu \mu^{m} \frac{\partial^{2 m}}{\partial x^{2 m}}\right|_{x=c_{k}}\left(1-x^{2}\right)^{-m-\frac{1}{2}} \Pi_{k}(x)^{m+1}\left(\mu \Pi_{k}(x)+2 G_{k}(x)\right)^{m}
\end{aligned}
$$

Here, the $x$-integral was evaluated by residue methods. Formula (2.15) provides an expansion to all orders in $P_{k}$ of the dual quantum order parameters $a_{D k}$. To evaluate the 
expansion coefficients, it will prove convenient to have the following derivative terms

$$
\begin{aligned}
G_{k}\left(c_{k}\right) & =-s_{k}^{2} \\
G_{k}^{\prime}\left(c_{k}\right) & =\frac{3}{2} c_{k} \\
G_{k}^{\prime \prime}\left(c_{k}\right) & =\left(\frac{2}{3} N^{2} s_{k}^{2}+\frac{4}{3} s_{k}^{2}+\frac{1}{2} c_{k}^{2}\right) \frac{1}{s_{k}^{2}} \\
G_{k}^{\prime \prime \prime}\left(c_{k}\right) & =\left(-N^{2} s_{k}^{2}+\frac{3}{2} c_{k}^{2}+\frac{7}{4} s_{k}^{2}\right) \frac{c_{k}}{s_{k}^{4}}
\end{aligned}
$$

as well as the fact that $\Pi_{k}\left(c_{k}\right)=-(-)^{k} s_{k}^{2} P_{k}$.

\section{(c) Contributions to second order}

We shall now use the above expression to evaluate contributions to second order in $P_{k}$ first, and find that

$$
\begin{aligned}
\left.a_{D k}^{(1)}\right|_{\text {quadratic in } P_{k}=-}=\frac{i}{2 s_{k} N^{3}}[ & \left(-\frac{5}{4} c_{k}^{2}+\frac{2}{3} s_{k}^{2}-\frac{3}{2}+\frac{1}{3} N^{2} s_{k}^{2}\right) P_{k}^{2}-\Pi_{k}^{\prime}\left(c_{k}\right)^{2} \\
& \left.+(-)^{k} s_{k}^{2} P_{k} \Pi_{k}^{\prime \prime}\left(c_{k}\right)+3(-)^{k} c_{k} P_{k} \Pi_{k}^{\prime}\left(c_{k}\right)\right]
\end{aligned}
$$

The derivatives are easily evaluated and given as follows

$$
\begin{aligned}
& \Pi_{k}^{\prime}\left(c_{k}\right)=-\sum_{l \neq k}^{N-1}(-)^{l} \frac{s_{l}^{2}}{c_{k}-c_{l}} P_{l} \\
& \Pi_{k}^{\prime \prime}\left(c_{k}\right)=2 \sum_{l \neq k}^{N-1}(-)^{l} \frac{s_{l}^{2}}{\left(c_{k}-c_{l}\right)^{2}} P_{l}
\end{aligned}
$$

Higher order terms may be calculated analogously, but we shall not need them here. Instead, we shall use some shortcuts and compute directly the beta function.

\section{THE BETA FUNCTION AND THE PREPOTENTIAL}

The renormalization group beta function $u$, discussed in (2.3) is obtained in terms of the leading coefficient multiplying $x^{N-2}$ of $P(x)$. Remarkably, this quantity also assumes a simple expression in terms of the coordinates $P_{k}$, and we find

$$
u=-4 \Lambda^{2} \tilde{u} ; \quad \tilde{u}=\frac{1}{N} \sum_{k=1}^{N-1}(-)^{k} s_{k}^{2} P_{k}
$$

Here, we have dropped a constant contribution $\left(-N \Lambda^{2}\right)$ to $u$, since it would yield just a constant shift of the prepotential $\mathcal{F}$ which is physically immaterial. 
Now, instead of calculating out the expansions of $a_{D k}$ in terms of the classical variables $P_{k}$, then inverting to obtain an expansion of $P_{k}$ in terms of $a_{D k}$, and finally inserting the expansions of $P_{k}$ into (2.19) to obtain the beta function $u$, it is much more advantageous to exhibit the summation that occurs in $\tilde{u}$ before effecting the substitution. In particular, this procedure will avoid having to perform major resummations which would have to be carried out by contour integrations anyway. Thus, we shall instead consider the following sum which directly exhibits $\tilde{u}$

$$
\tilde{u}=-i \sum_{k=1}^{N-1} s_{k} a_{D k}+\sum_{m=1}^{\infty} \alpha_{D}^{(m)} \quad \alpha_{D}^{(m)}=i \sum_{k=1}^{N-1} s_{k} a_{D k}^{(m)}
$$

The resummed expansion parameters $\alpha_{D}^{(m)}$ may be expressed in terms of the functions $\Pi(x)$ and $G(x)$, defined in (2.14), and are given by

$$
\alpha_{D}^{(m)}=\sum_{k=1}^{N-1} \frac{\Gamma\left(m+\frac{1}{2}\right)}{\Gamma\left(\frac{1}{2}\right) m ! N^{2 m+1}} \int_{0}^{1} d \mu \mu^{m} \oint_{B_{k}} \frac{d x}{2 \pi i} \frac{s_{k}}{\left(1-x^{2}\right)^{m+\frac{1}{2}}} \Pi(x)^{m+1}(\mu \Pi(x)+2 G(x))^{m}
$$

What prevents us from carrying out the sum over $k$ by connecting the various contours $B_{k}$ in the above formula is twofold. First, the square root would force us to perform a line integration around the lines from \pm 1 to $\infty$. This would not represent an advantage over a direct evaluation with residue methods. Second, the factor $s_{k}$ depends upon $k$, so we cannot just add up the various contours $B_{k}$.

\section{(a) Combining contours}

We can solve both problems by replacing $s_{k}$ inside the above integrand by a $k$ independent function that takes the value $s_{k}$ at $x=c_{k}$ for all $k=1, \cdots, N-1$, but whose derivatives of orders $1, \cdots, 2 m$ vanish at this point. Replacing $s_{k}$ by such a function inside (3.3) for each value of $m$ will not alter the value of the integral. It is very easy to obtain such a function by expanding the value at $c_{k}$ in a Taylor series around $x$ instead. For example, defining $s(x)=\sqrt{1-x^{2}}$, we have

$$
s_{k}=s\left(c_{k}\right)=s(x)+\left(c_{k}-x\right) s^{\prime}(x)+\frac{1}{2}\left(c_{k}-x\right)^{2} s^{\prime \prime}(x)+\mathcal{O}\left(\left(c_{k}-x\right)^{2}\right)
$$

To order $m=1$, the expressions we need are given by

$$
\frac{s_{k}}{\left(1-x^{2}\right)^{3 / 2}}=\frac{1}{1-x^{2}}+\left(x-c_{k}\right) \frac{x}{\left(1-x^{2}\right)^{2}}-\frac{1}{2}\left(x-c_{k}\right)^{2} \frac{1}{\left(1-x^{2}\right)^{3}}+\mathcal{O}\left(\left(x-c_{k}\right)^{3}\right)
$$

while to order $m=2$, we have

$$
\begin{aligned}
\frac{s_{k}}{\left(1-x^{2}\right)^{5 / 2}} G(x)^{2}= & \frac{G(x)^{2}}{\left(1-x^{2}\right)^{2}}-\frac{x}{\left(1-x^{2}\right)^{2}} G(x)-\frac{1}{2} \frac{1}{1-x^{2}}-\frac{c_{k}}{6 s_{k}^{4}}\left(1+2 N^{2}\right)\left(x-c_{k}\right) \\
& -\frac{1}{24 s_{k}^{6}}\left(3+11 c_{k}^{2}+28 N^{2} c_{k}^{2}\right)\left(x-c_{k}\right)^{2}+\mathcal{O}\left(\left(x-c_{k}\right)^{3}\right)
\end{aligned}
$$


Inside the integrals, the $\mathcal{O}()$ terms vanish identically and may be dropped. What we have gained is that the first term in (3.5) and the first three terms in (3.6) are $k$-independent and various contours in the sum (3.3) may now be collected together. As can be established by inspecting (3.3), (3.5) and (3.6), the only poles other than those occuring at $c_{k}, k=$ $1, \cdots, N-1$ are the poles at $x= \pm 1$. Thus, we have for those parts of the integral

$$
\sum_{k=1}^{N-1} \oint_{B_{k}}=-\oint_{ \pm 1}
$$

The remaining terms are handled by standard residue calculations.

(b) Contributions for $m=1$

Substituting (3.5) into (3.3) for $m=1$, we find, using also (3.7)

$$
\begin{aligned}
\alpha_{D}^{(1)}= & -\frac{1}{2 N^{3}} \oint_{ \pm 1} \frac{d x}{2 \pi i} \frac{1}{1-x^{2}}\left(\frac{1}{3} \Pi(x)^{3}+\Pi(x)^{2} G(x)\right) \\
& +\frac{1}{2 N^{3}} \sum_{k=1}^{N-1} \oint_{B_{k}} \frac{d x}{2 \pi i}\left\{x\left(1-x^{2}\right)-\frac{1}{2}\left(x-c_{k}\right)\right\} \frac{\frac{1}{3} \Pi_{k}(x)^{3}+\Pi_{k}(x)^{2} G_{k}(x)}{\left(x-c_{k}\right)^{2}\left(1-x^{2}\right)^{3}}
\end{aligned}
$$

It is straightforward to carry out the residue calculations at \pm 1 in the first integrals, and at $c_{k}, k=1, \cdots, N-1$ in the second integrals. Using also the fact that $G( \pm 1)= \pm 1$, we find

$$
\begin{aligned}
\alpha_{D}^{(1)}= & +\frac{1}{4 N^{3}}\left\{\Pi(1)^{2}+\Pi(-1)^{2}+\frac{1}{3} \Pi(1)^{3}-\frac{1}{3} \Pi(-1)^{3}\right\} \\
& -\frac{1}{4 N^{3}} \sum_{k=1}^{N-1}\left\{\left(1+3 c_{k}^{2}\right) P_{k}^{2}-4(-)^{k} c_{k} P_{k} \Pi_{k}^{\prime}\left(c_{k}\right)+(-)^{k}\left(\frac{1}{3}+2 c_{k}^{2}\right) P_{k}^{3}-2 c_{k} P_{k}^{2} \Pi_{k}^{\prime}\left(c_{k}\right)\right\}
\end{aligned}
$$

Notice that the combinations $\Pi( \pm 1)$ are simply given by

$$
\Pi( \pm 1)=-\sum_{k=1}^{N-1}(-)^{k}\left(c_{k} \pm 1\right) P_{k}
$$

Making use of expression (2.18) for $\Pi_{k}^{\prime}\left(c_{k}\right)$, and some straightforward trigonometric identities, we find

$$
\begin{aligned}
\alpha_{D}^{(1)}= & \frac{1}{4 N^{3}} \sum_{k=1}^{N-1} s_{k}^{2} P_{k}^{2}+\frac{1}{12 N^{3}}\left(\Pi(1)^{3}-\Pi(-1)^{3}\right) \\
& -\frac{1}{4 N^{3}} \sum_{k=1}^{N-1}\left\{(-)^{k}\left(\frac{1}{3}+2 c_{k}^{2}\right) P_{k}^{3}-2 c_{k} P_{k}^{2} \Pi_{k}^{\prime}\left(c_{k}\right)\right\}
\end{aligned}
$$


Notice that the quadratic part is diagonal in $P_{k}$.

\section{(c) Contributions for $m=2$}

In a manner analogous to the computation of the $m=1$ contributions, we get those for $m=2$ by substituting (3.6) into (3.3) for $m=2$. Using the fact that $G^{\prime}( \pm 1)=\frac{1}{3}\left(2 N^{2}+1\right)$, we find

$$
\begin{aligned}
\alpha_{D}^{(2)}= & -\frac{1}{24 N^{5}}\left(2 N^{2}+1\right)\left(\Pi(1)^{3}-\Pi(-1)^{3}\right) \\
& -\frac{1}{N^{5}} \sum_{k=1}^{N-1}\left\{c_{k}\left(\frac{1}{4}+\frac{1}{2} N^{2}\right) P_{k}^{2} \Pi_{k}^{\prime}\left(c_{k}\right)-(-)^{k}\left(\frac{1}{16}+\frac{11}{48} c_{k}^{2}+\frac{7}{12} N^{2} c_{k}^{2}\right) P_{k}^{3}\right\}
\end{aligned}
$$

\section{(d) Expressing $P_{k}$ and the beta function in terms of $a_{D k}$}

To obtain the beta function to cubic order in $a_{D k}$, it suffices to substitute into (3.11) and (3.12) the expansion of $P_{k}$ in terms of $a_{D k}$ up to quadratic order only. This inversion is easily obtained from (2.11) and (2.17), and we find

$$
\begin{aligned}
P_{k}=-(-)^{k} \frac{i N}{s_{k}} a_{D k}-\frac{(-)^{k}}{2 s_{k}^{4}}[ & \left(-\frac{5}{4} c_{k}^{2}+\frac{2}{3} s_{k}^{2}-\frac{3}{2}+\frac{1}{3} N^{2} s_{k}^{2}\right) a_{D k}^{2}+\frac{s_{k}^{2}}{N^{2}} \Pi_{k}^{\prime}\left(c_{k}\right)^{2} \\
& \left.+i \frac{s_{k}^{3}}{N} a_{D k} \Pi_{k}^{\prime \prime}\left(c_{k}\right)+\frac{3 i}{N} s_{k} c_{k} a_{D k} \Pi_{k}^{\prime}\left(c_{k}\right)\right]+\mathcal{O}\left(a_{D k}^{3}\right)
\end{aligned}
$$

Putting all together, we find for the beta function the following expression

$$
\begin{aligned}
\tilde{u}= & -i \sum_{k=1}^{N-1} s_{k} a_{D k}-\frac{1}{4 N} \sum_{k=1}^{N-1} a_{D k}^{2}-\frac{1}{24 N^{5}}\left(\Pi(1)^{3}-\Pi(-1)^{3}\right) \\
& -\frac{i}{4 N^{2}} \sum_{k=1}^{N-1} \frac{1}{s_{k}^{3}}\left[\left(\frac{7}{12}+c_{k}^{2}\right) a_{D k}^{3}-\frac{s_{k}^{2}}{N^{2}} a_{D k} \Pi_{k}^{\prime}\left(c_{k}\right)^{2}-i \frac{s_{k}^{3}}{N} a_{D k}^{2} \Pi_{k}^{\prime \prime}\left(c_{k}\right)-\frac{2 i}{N} c_{k} s_{k} a_{D k}^{2} \Pi_{k}^{\prime}\left(c_{k}\right)\right]
\end{aligned}
$$

Here, the derivatives of $\Pi_{k}$ are evaluated as follows

$$
\begin{aligned}
\Pi_{k}^{\prime}\left(c_{k}\right) & =i N \sum_{l \neq k} \frac{s_{l} a_{D l}}{c_{k}-c_{l}} \\
\Pi_{k}^{\prime \prime}\left(c_{k}\right) & =-2 i N \sum_{l \neq k} \frac{s_{l} a_{D l}}{\left(c_{k}-c_{l}\right)^{2}}
\end{aligned}
$$

It remains to rearrange the sum in the last line of $(3.14)$, and to combine with the $\Pi(+1)^{3}-$ $\Pi(-1)^{3}$ term of the first line. The result is as follows

$$
\tilde{u}=-i \sum_{k=1}^{N-1} s_{k} a_{D k}-\frac{1}{4 N} \sum_{k=1}^{N-1} a_{D k}^{2}-\frac{i}{16 N^{2}}\left[\sum_{k=1}^{N-1} \frac{a_{D k}^{3}}{s_{k}^{3}}-4 \sum_{k \neq l}^{N-1} \frac{a_{D k}^{2} a_{D l} s_{l}}{\left(c_{k}-c_{l}\right)^{2}}\right]
$$


Restoring the original $\Lambda$-dependence, we now easily find the renormalization group beta function

$$
u=2 i \Lambda \sum_{k=1}^{N-1} s_{k} a_{D k}+\frac{1}{4 N} \sum_{k=1}^{N-1} a_{D k}^{2}+\frac{i}{32 N^{2} \Lambda}\left[\sum_{k=1}^{N-1} \frac{a_{D k}^{3}}{s_{k}^{3}}-4 \sum_{k \neq l}^{N-1} \frac{a_{D k}^{2} a_{D l} s_{l}}{\left(c_{k}-c_{l}\right)^{2}}\right]
$$

\section{(e) The prepotential}

Starting from the result for the beta function in (3.17), and making use of (2.3), we obtain our final expression for the prepotential at strong coupling

$$
\begin{aligned}
\mathcal{F}\left(a_{D}\right)=-\frac{2 N \Lambda}{\pi} & \sum_{k=1}^{N-1} s_{k} a_{D k}-\frac{i}{4 \pi} \sum_{k=1}^{N-1} a_{D k}^{2} \log \frac{a_{D k}}{\Lambda_{k}} \\
& +\frac{1}{32 \pi N \Lambda}\left[\sum_{k=1}^{N-1} \frac{a_{D k}^{3}}{s_{k}^{3}}-4 \sum_{k \neq l}^{N-1} \frac{a_{D k}^{2} a_{D l} s_{l}}{\left(c_{k}-c_{l}\right)^{2}}\right]
\end{aligned}
$$

Here, the values of the normalizations $\Lambda_{k}$ are not available directly from the integration of the renormalization group equation in (2.3). (In principle, the renormalization group equation will determine the prepotential $\mathcal{F}$ through (2.3) only up to a homogeneous function of degree 2 in $a_{D k}$. Here, however, we are performing an expansion in powers of $a_{D k} / \Lambda$, and we know what that expansion looks like from the evaluation of the $A_{k}$ periods. In particular, only a single logarithmic term will appear. Thus, knowing the form of the expansions of both $u$ and $\mathcal{F}$, the only remaining indeterminacy of the renormalization group equation are terms quadratic in $a_{D k}$.) They can be obtained from a direct calculation to that order of the $A_{k}$ periods, a calculation that has already been carried out by Douglas and Shenker [11]. We shall quote their results here :

$$
\log \frac{\Lambda_{k}}{\Lambda}=\frac{3}{2}+\log s_{k}
$$

\section{(f) Comparison with known results}

For $\mathrm{SU}(2), \mathrm{N}=2$, we have $s_{1}=1$ and thus

$$
\mathcal{F}=-\frac{4 \Lambda}{\pi} a_{D}-\frac{i}{4 \pi} a_{D}^{2} \log \frac{a_{D}}{\Lambda}+\frac{1}{64 \pi \Lambda} a_{D}^{3}
$$

which agrees with $[1,8]$ after suitably adjusting for the slightly different definitions of $\Lambda$. For $\mathrm{SU}(3), \mathrm{N}=3$, we have $s_{1}=s_{2}=\sqrt{3} / 2$ and $c_{1}=-c_{2}=1 / 2$ and thus

$$
\begin{aligned}
\mathcal{F}= & -\frac{3 \sqrt{3} \Lambda}{\pi}\left(a_{D 1}+a_{D 2}\right)-\frac{i}{4 \pi}\left(a_{D 1}^{2} \log \frac{a_{D 1}}{\Lambda_{1}}+a_{D 2}^{2} \log \frac{a_{D 2}}{\Lambda_{2}}\right) \\
& +\frac{1}{144 \sqrt{3} \pi \Lambda}\left(a_{D 1}+a_{D 2}\right)\left(4 a_{D 1}^{2}-13 a_{D 1} a_{D 2}+4 a_{D 2}^{2}\right)
\end{aligned}
$$


Again, taking into account the difference in definition of $\Lambda$ with [8], we find agreement. Finally, for all SU(N), the logarithmic terms were computed in [11], and the normalization of the logs agrees.

\section{(g) Physical interpretation}

To understand more clearly the physical origin of the non-perturbative correction terms that we have calculated, it is helpful to derive, within this approximation, the (dual gauge) coupling constant $\tau_{m n}^{D}=\partial^{2} \mathcal{F} / \partial a_{D m} \partial a_{D n}$. We find

$$
\begin{aligned}
\tau_{m m}^{D} & =-\frac{i}{2 \pi} \log \frac{a_{D m}}{\Lambda_{m}}-\frac{3 i}{4 \pi}+\frac{3}{16 \pi N \Lambda} \frac{a_{D m}}{s_{m}^{3}}-\frac{1}{8 \pi N \Lambda} \sum_{l \neq m} \frac{2 a_{D l} s_{l}}{\left(c_{m}-c_{l}\right)^{2}} \\
\tau_{m n} & =-\frac{1}{4 \pi N \Lambda} \frac{a_{D m} s_{n}+a_{D n} s_{m}}{\left(c_{m}-c_{n}\right)^{2}}
\end{aligned}
$$

Identifying $\tau_{m m}^{D}$ in terms of the dual coupling constant $g_{D m}$ and dual instanton angle $\theta_{D m}$, as usual, we find that the cubic correction terms in the prepotential have a dependence on $g_{D m}$ and $\theta_{D m}$ through

$$
e^{2 \pi i \tau_{m m}^{D}}=e^{-\frac{8 \pi^{2}}{g_{D m}^{2}}+i \theta_{D m}}
$$

suggesting that the effects are due to (magnetic sector) instantons. It would be very illu-

minating to see how the same effects can be identified from the physics of light monopoles near the strong coupling points.

\section{REFERENCES}

[1] N. Seiberg and E. Witten, Nucl. Phys. B 426 (1994) 19, hep-th/9407087;

[2] A. Klemm, W. Lerche, S. Yankielowicz, and S. Theisen, Phys. Lett. B 344 (1995) 169 ;

P.C. Argyres and A. Faraggi, Phys. Rev. Lett. 73 (1995) 3931;

[3] N. Seiberg and E. Witten, Nucl. Phys. B 431 (1994) 484, hep-th/9408099;

A. Hanany and Y. Oz, Nucl. Phys. B452 (1995) 73, hep-th/9505075;

P.C. Argyres and A. Shapere, Nucl. Phys. B 461 (1996) 437, hep-th/9609175;

A. Hanany, Nucl. Phys. B 466 (1996) 85, hep-th/9509176.

[4] P.C. Argyres, R. Plesser, and A. Shapere, Phys. Rev. Lett. 75 (1995) 1699, hepth/9505100;

J. Minahan and D. Nemeshansky, Nucl. Phys. B 464 (1996) 3, hep-th/9507032;

U.H. Danielsson and B. Sundborg, Phys. Lett. B 358 (1995) 273; hep-th/9504102;

A. Brandhuber and K. Landsteiner, Phys. Lett. B 358 (1995) 73, hep-th/9507008;

M. Alishahiha, F. Ardalan, and F. Mansouri, Phys. Lett. B 381 (1996) 446, hepth/9512005. 
[5] N. Dorey, V. Khoze, and M. Mattis, Phys. Rev. D 54 (1996) 7832, hep-th/9606199; Phys. Lett. B 388 (1996) 324, hep-th/9607066; hep-th/9607202;

Y. Ohta, hep-th/9604051, hep-th/9604059;

D. Finnell and P. Pouliot, Nucl. Phys. B 453 (1995) 225;

A. Yung, hep-th/9605096;

F. Fucito and G. Travaglini, hep-th/9605215

H. Aoyama, T. Harano, M. Sato, and S. Wada, hep-th/9607076;

T. Harano and M. Sato, hep-th/9608060

[6] E. D'Hoker, I.M. Krichever, and D.H. Phong, hep-th/9609041; hep-th/9609145, to appear in Nucl. Phys. B;

[7] P. Argyres and M. Douglas, Nucl. Phys. B 448 (1995) 93, hep-th/9505062.

[8] A. Klemm, W. Lerche, and S. Theisen, Int. J. Mod. Phys. A 11 (1996) 1929, hepth/9505150;

K. Ito and S.K. Yang, Phys. Rev. D 53 (1996) 2213, hep-th/9603073;

J.M. Isidro, A. Mukherjee, J.P. Nunes, and H.J. Schnitzer, hep-th/9609116.

[9] M. Matone, Phys. Lett. B 357 (1995) 342;

G. Bonelli and M. Matone, Phys. Rev. Lett. 76 (1996) 4107;

G. Bonelli and M. Matone, hep-th/9605090;

T. Eguchi and S.K. Yang, Mod. Phys. Lett. A 11 (1996) 131;

P. Howe and P. West, hep-th/9607239

J. Sonnenschein, S. Theisen, and S. Yankielowicz, Phys. Lett. B 367 (1996) 145, hep-th/9510129.

[10] E. D'Hoker, I.M. Krichever and D.H. Phong, hep-th/9610156.

[11] M. Douglas and S. Shenker, Nucl. Phys. B 448 (1995) 271, hep-th/9503163. 